\title{
Proteomic profiling of leukocytes reveals dysregulation of adhesion and integrin proteins in chronic kidney disease-related atherosclerosis
}

Joanna Tracz ${ }^{1}$, Luiza Handschuh ${ }^{1}$, Maciej Lalowski ${ }^{1,2}$, Łukasz Marczak ${ }^{1}$, Katarzyna KostkaJeziorny $^{3}$, Bartłomiej Perek ${ }^{4}$, Maria Wanic-Kossowska ${ }^{5}$, Alina Podkowińska ${ }^{6}$, Andrzej Tykarski $^{3}$, Dorota Formanowicz ${ }^{7}$, Magdalena Luczak ${ }^{1 *}$

${ }^{1}$ Institute of Bioorganic Chemistry, Polish Academy of Sciences, Noskowskiego 12/14, 61-704 Poznan, Poland;

${ }^{2}$ Helsinki Institute for Life Science (HiLIFE) and Faculty of Medicine, Biochemistry/Developmental Biology, Meilahti Clinical Proteomics Core Facility, University of Helsinki

${ }^{3}$ Department of Hypertension, Angiology and Internal Disease, Poznan University of Medical Sciences, Długa 1/2, 61-848, Poznan, Poland;

${ }^{4}$ Department of Cardiac Surgery and Transplantology, Poznan University of Medical Sciences, Długa 1/2, 61848, Poznan, Poland;

${ }^{5}$ Department of Nephrology, Transplantology and Internal Medicine, Poznan University of Medical Sciences, Przybyszewskiego 49, 60-355 Poznan, Poland;

${ }^{6}$ Dialysis station Dravis sp. z o.o., Dojazd 34, 60-631 Poznan, Poland

${ }^{7}$ Chair and Department of Medical Chemistry and Laboratory Medicine, Poznan University of Medical Sciences, Rokietnicka 8, 60-806, Poznan, Poland.

\section{TABLE OF CONTENTS}

The following supporting information is available free of charge at ACS website

http://pubs.acs.org

\section{List of Figures}

\section{Figure S1}

Microscopy and flow cytometry analysis of isolated cells

\section{Figure S2}

Enrichment analysis in the context of compartmental localization

\section{Figure S3}

Unsupervised principal component analysis (PCA)

\section{Figure S4}

Heat maps presenting abundance of identified DEPs 


\section{Figure S5}

Correlation coefficient matrix for DEPs in CKD5 vs CVD2 comparison

\section{Figure S6}

IPA functional analysis

\section{Figure S7}

TLN1 immunoblot - entire membrane

\section{List of Tables}

\section{Table S1}

Pearson's correlation coefficients between the LFQ (label-free quantification) intensities from replicates in all experimental groups

\section{Table S2}

List of primers used in ddPCR analysis

\section{Table S3}

List of peptide transitions with precursor and product masses, charges, retention times and collision energies used for MRM analysis

\section{Table S4}

The results of correlation analyses between the identified DEPs and eGFR, the number of particular blood cells and leukocyte subpopulations

\section{Table S5}

Complete list of differentially expressed proteins (DEPs) identified in the study. The names of proteins, the number of identified peptides, p-values, fold changes, effect size for all group comparisons and UniProt accessions

\section{Table S6}

The results of Pearson's correlation analyses between the DEPs identified in CKD5 vs CVD2 comparison

\section{Table S7}

A detailed list of IPA annotation results for canonical pathway, diseases/functions and upstream regulator categories with $-\log _{10} \mathrm{~B}-\mathrm{H}$ corrected $\mathrm{p}$-values and $\mathrm{z}$-scores 
Figure S1: Microscopy and flow cytometry analysis of isolated cells

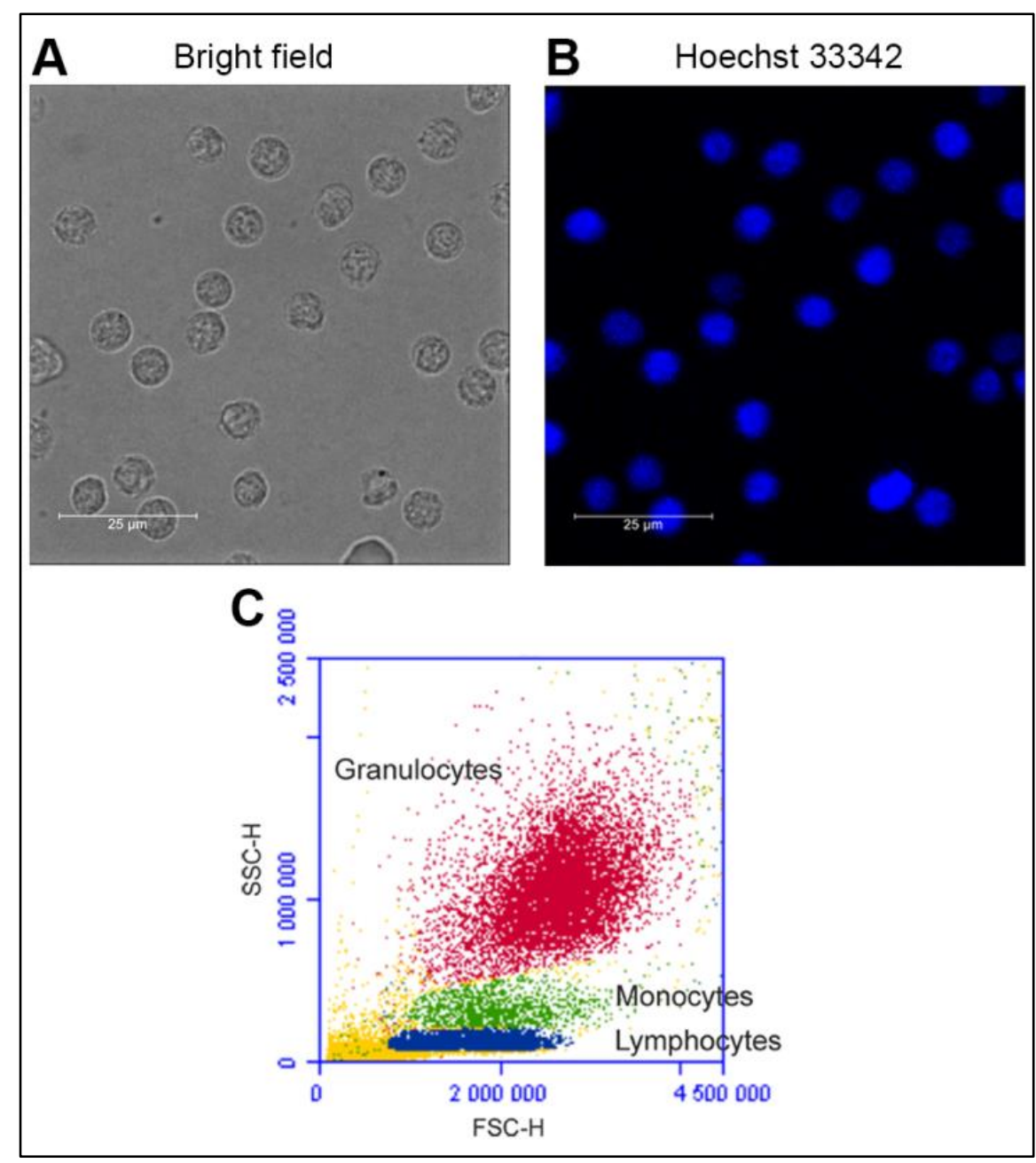

Isolated cells were evaluated using fluorescence microscopy and flow cytometry. Bright field (A) and fluorescence images of leukocytes after staining with Hoechst 33342 (B); (C) Gating of isolated leukocyte subpopulations by flow cytometry. 


\section{Figure S2}

Enrichment analysis in the context of compartmental localization
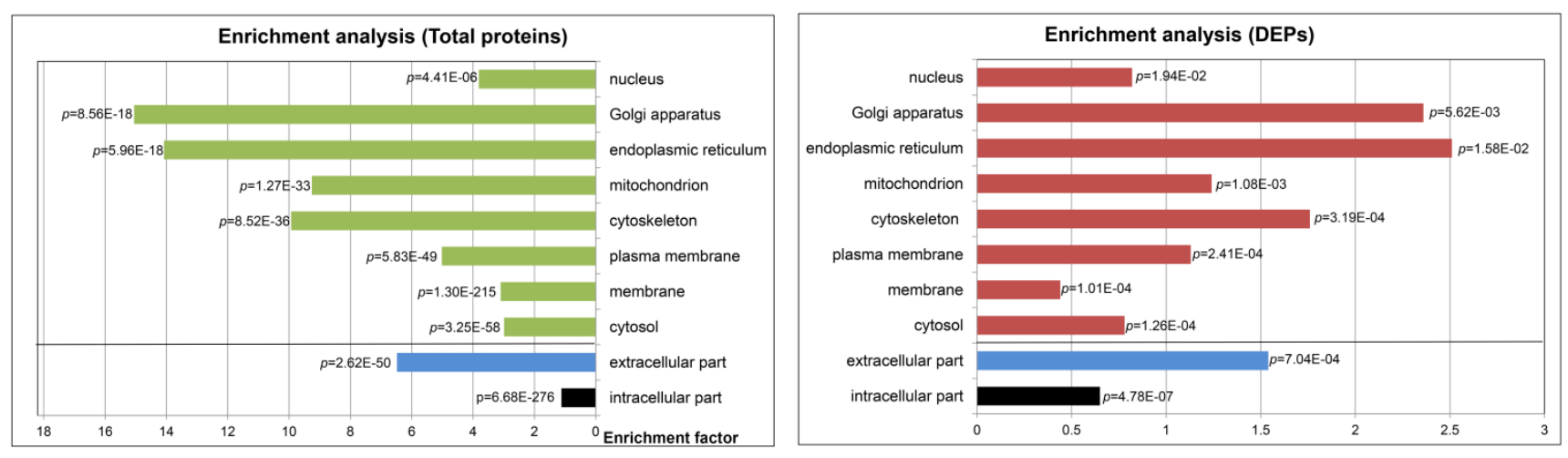

Enrichment analysis in the context of compartmental localization was performed for all identified proteins and DEPs. Fisher's exact with B-H correction at FDR of $2 \%$ was used. 


\section{Figure S3}

Unsupervised principal component analysis (PCA)

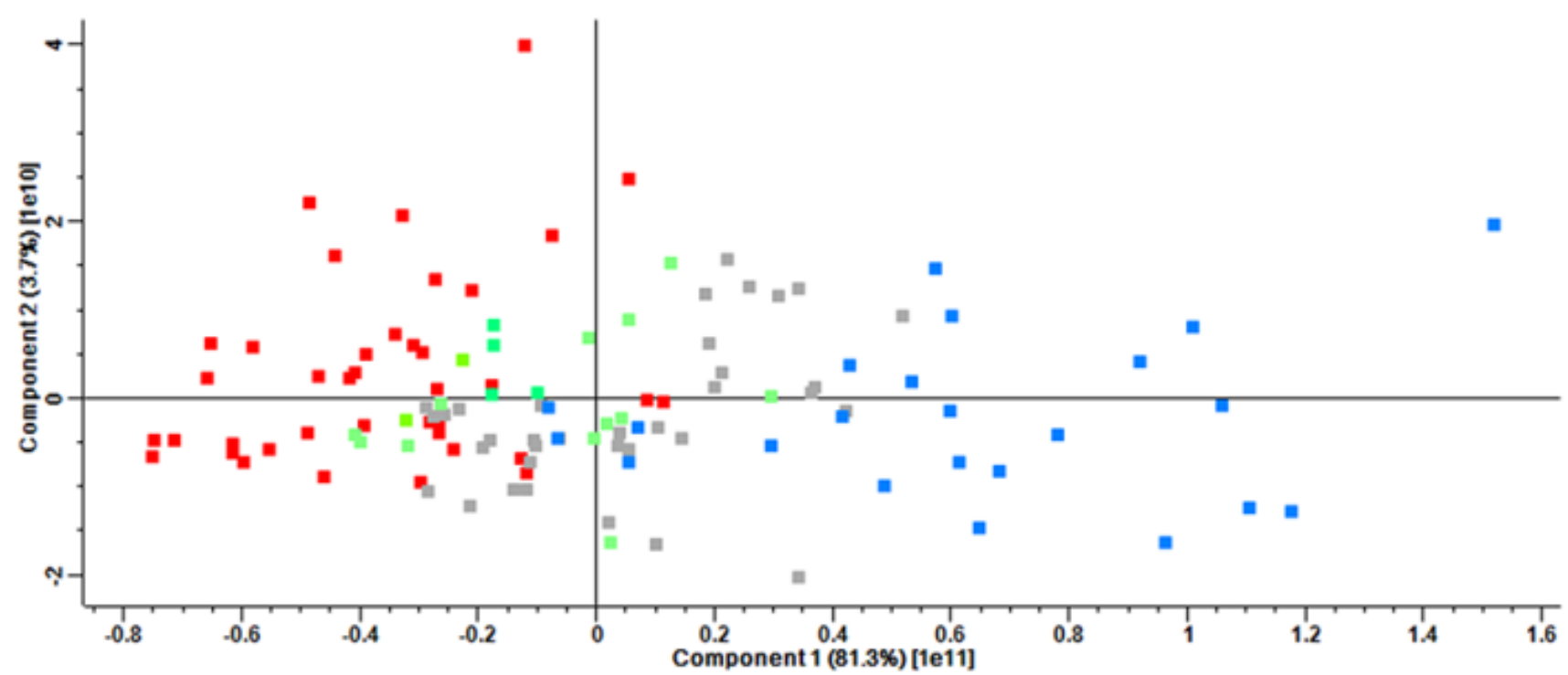

PCA performed on the total number of proteins identified in CKD5 (red), CVD2 (blue), CKD1-2 (green) and CVD1 (grey) experimental groups. 


\section{Figure S4}

Heat maps presenting abundance of identified DEPs

A

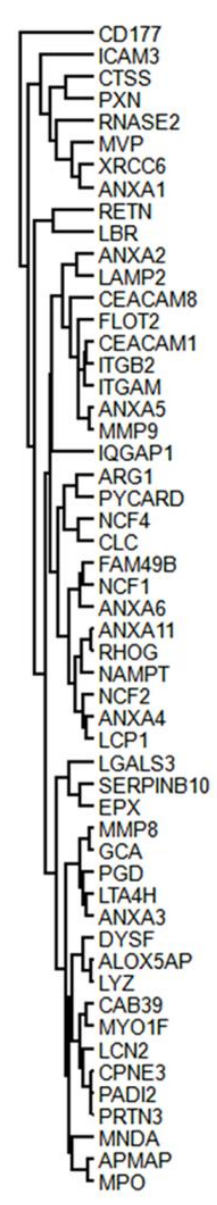

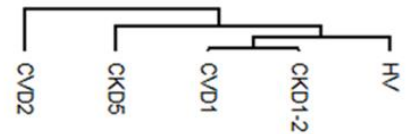

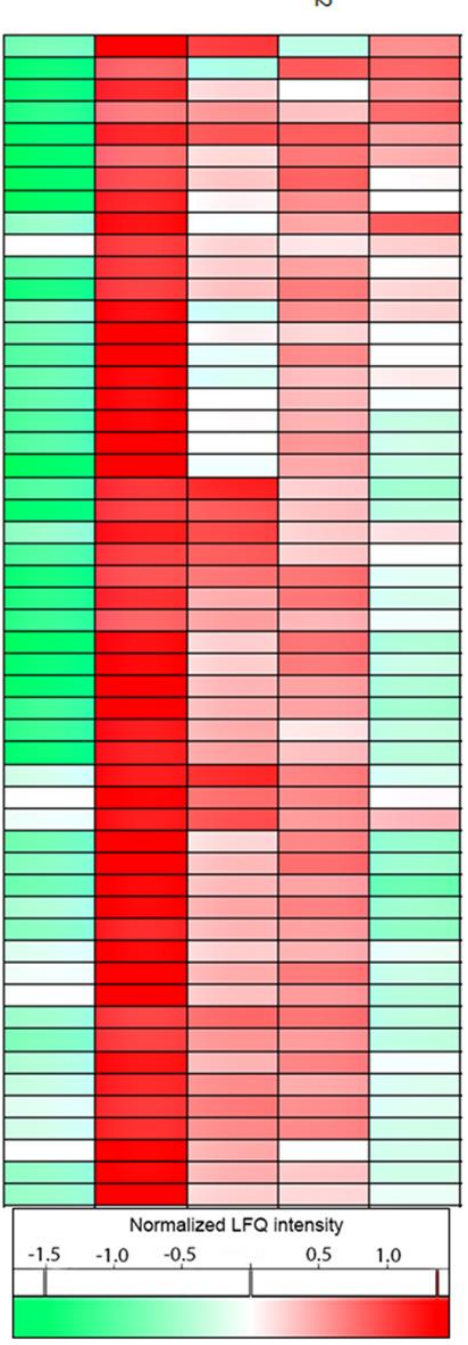

B

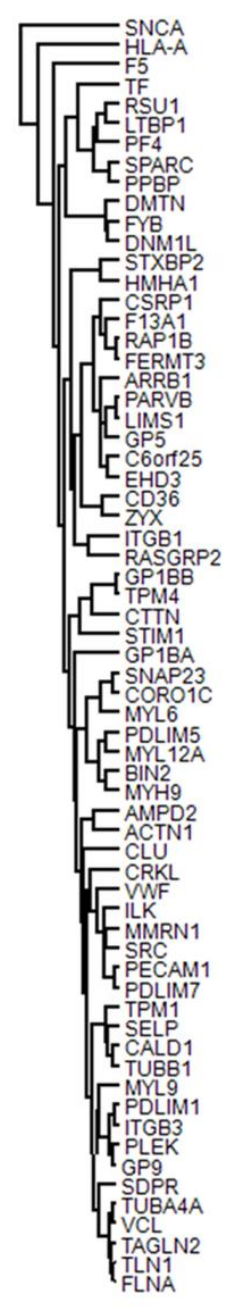

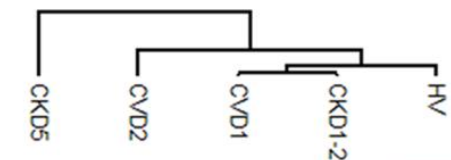

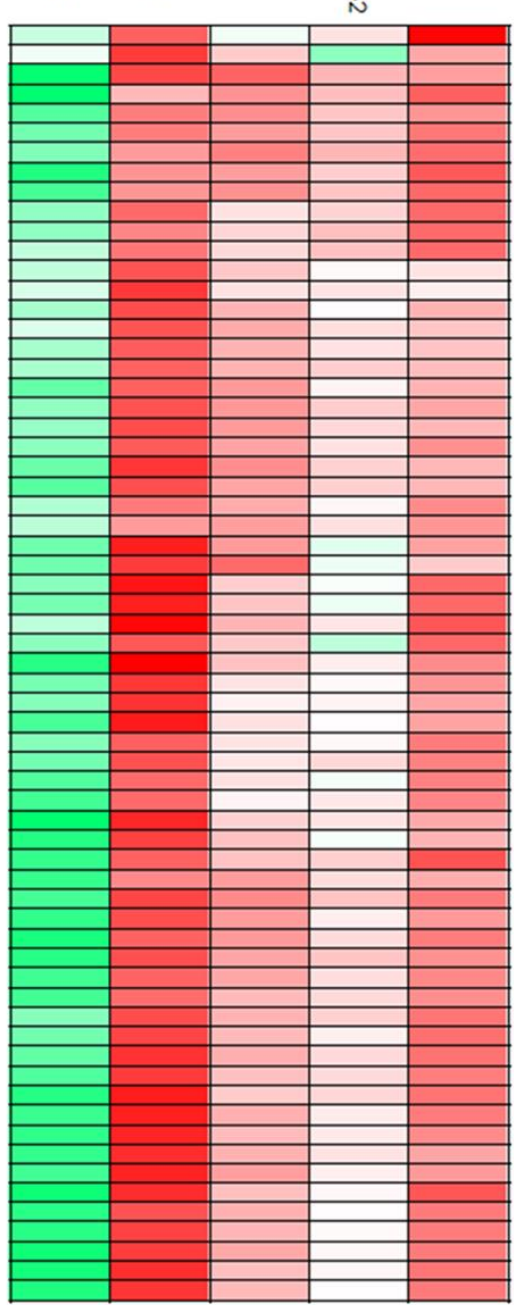

Heat maps presenting abundance of downregulated in CVD2 (A) or in CKD5 (B) proteins in the background of other experimental groups. 


\section{Figure S5}

Correlation coefficient matrix for DEPs in CKD5 vs CVD2 comparison

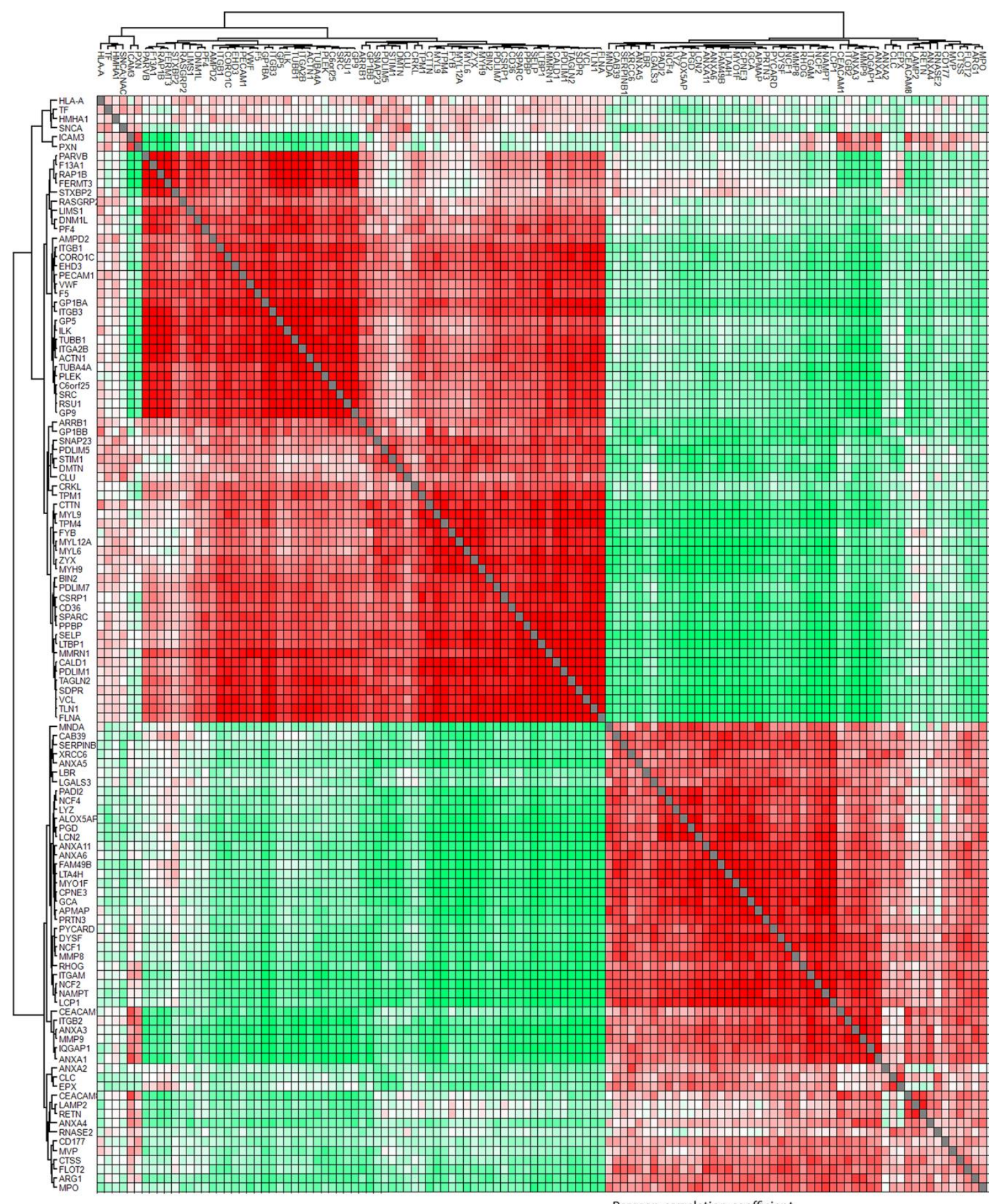

Pearson correlation coefficient

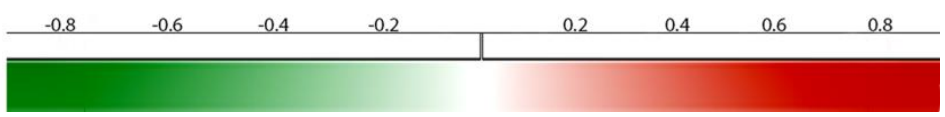

Heat map representation of Pearson's correlations for the abundances of DEPs identified in CKD5 vs CVD2 comparison. 


\section{Figure S6}

IPA functional analysis

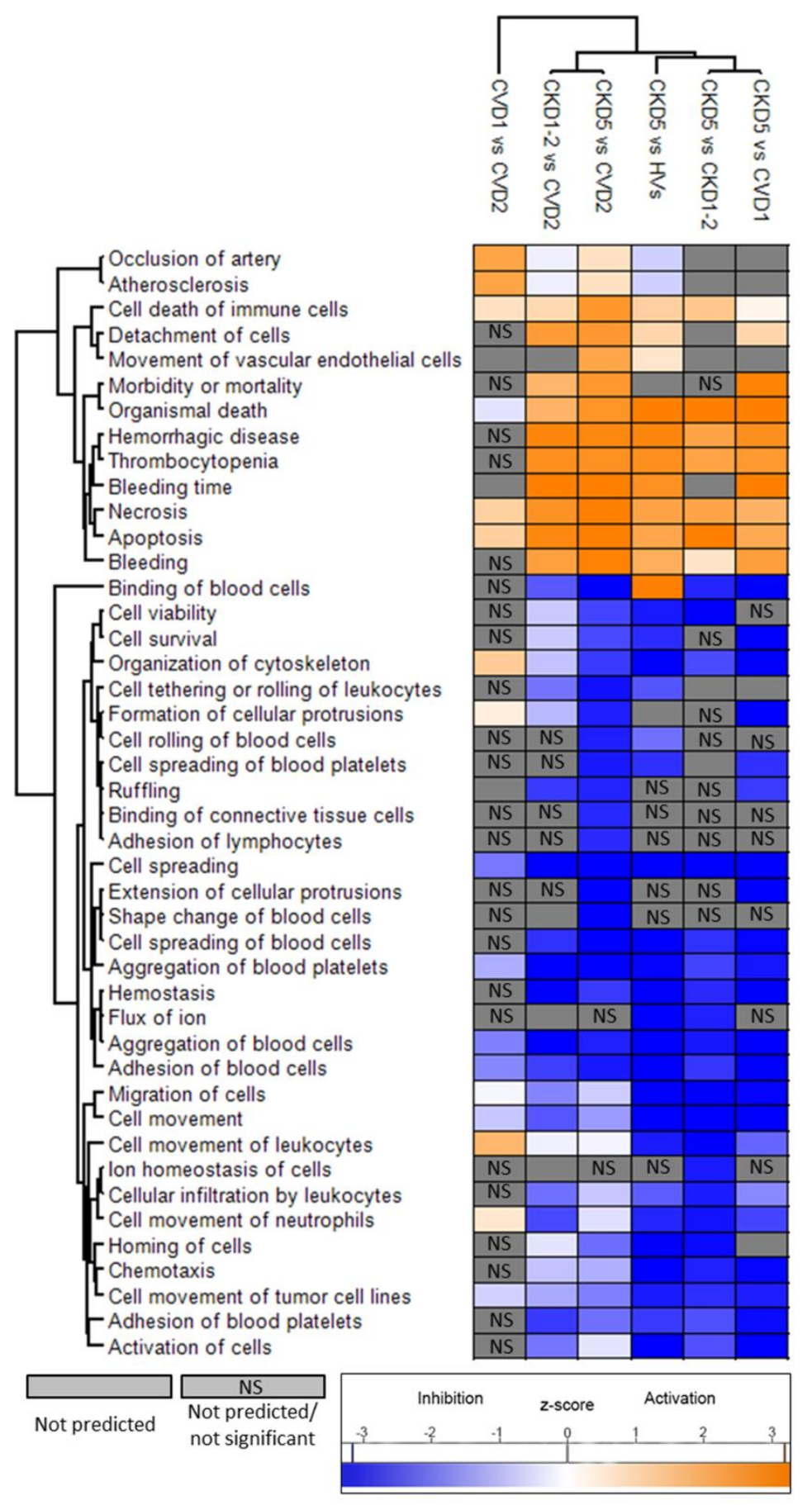

IPA functional analysis performed for 6 different comparisons. Categories with significant zscores are presented in blue inhibition; $\mathrm{z}$-score $\leq-2$ ) or orange (activation; $\mathrm{z}$-score $\geq 2$ ). Functions overrepresented according to B-H corrected p-values but without calculated $\mathrm{z}$ scores are presented in grey. Functions not enriched according to B-H corrected p-values are shown in grey and marked as NS. A detailed list of annotations with B-H p-values and zscores is presented in Table S7. 


\section{Figure S7}

TLN1 immunoblot - entire membranes

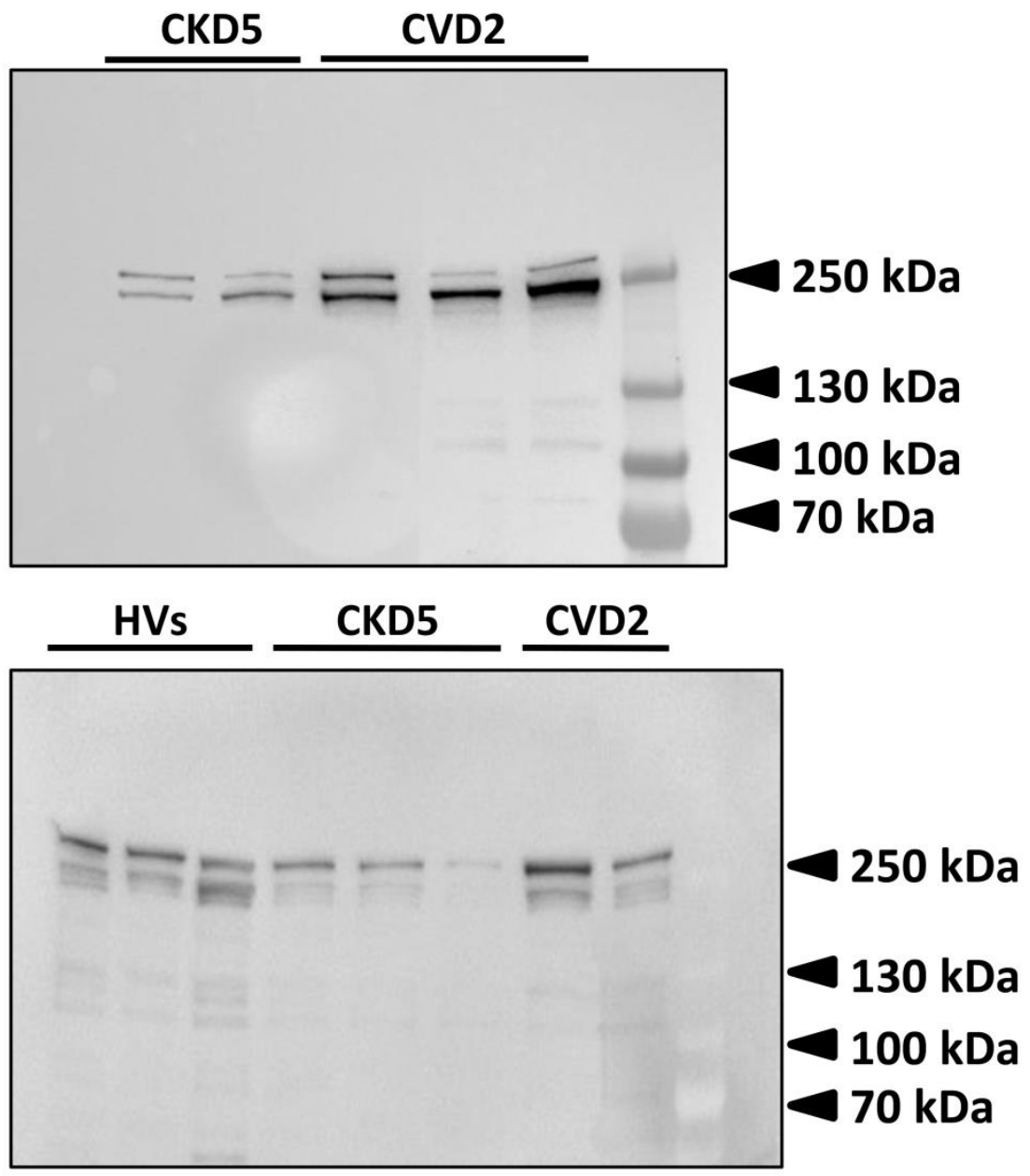

Entire membranes for the Western blot in Figure 3. $30 \mu \mathrm{g}$ of proteins were separated by 4$15 \%$ SDS-PAGE. Blots were incubated with the anti- TLN1 primary antibodies. Chemiluminescent detection was performed using the ChemiDoc XRS imaging system 